\title{
CORRECTION
}

\section{Author Correction: Cryo-EM structure and biochemical analysis reveal the basis of the functional difference between human PI3KC3-C1 and -C2}

Meisheng Ma, Jun-Jie Liu, Yan Li, Yuwei Huang, Na Ta, Yang Chen, Hua Fu, Ming-Da Ye, Yuehe Ding, Weijiao Huang, Jia Wang, Meng-Qiu Dong, Li Yu and Hong-Wei Wang

Cell Research (2020) 30:551-552; https://doi.org/10.1038/s41422-020-0311-z

Correction to: Cell Research https://doi.org/10.1038/cr.2017.94, published online 21 July 2017

We apologize for errors that we inadvertently introduced in Fig. $4 \mathrm{C}$ and Supplementary information, Figs. S5F, S4E and S6C, of the paper published online on July 21 2017. The bands of "a-P150_C2-BATs" in
Fig. 4C, "a-Beclin1 C1" in Supplementary information, Fig. S5F, and "a-P150_C2+6\%PI(4,5)P2" in Supplementary information, Fig. S6C were incorrectly cropped from the original western blot images. The labeling of "a-VPS34_VPS34 $\triangle$ CTD" and "a-VPS34_ATG14L $\triangle$ BATs" in Supplementary information, Fig. S4E were flipped. The corrected figures are provided below.

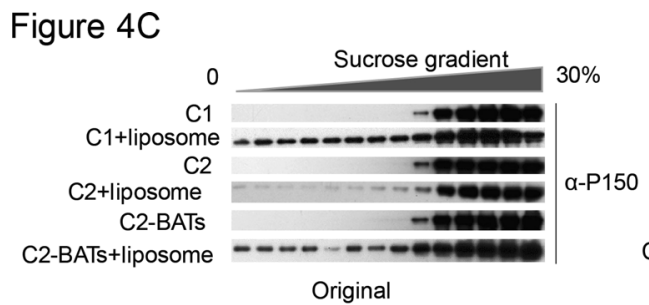

Figure S4E

ATG14L $\triangle$ BATS
VPS34 $\triangle$ CTD

Original

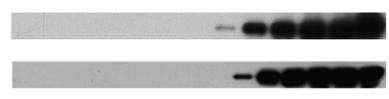

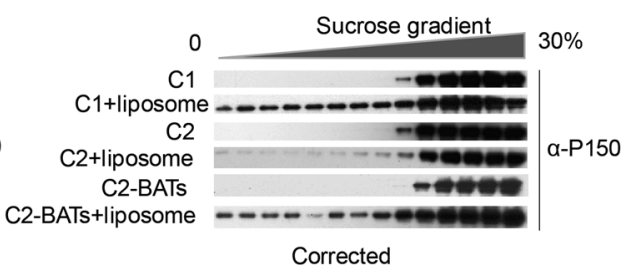

Corrected

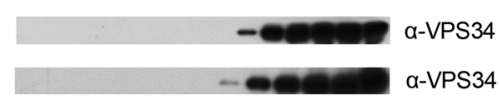

a-VPS34

Figure S5F

C1

a-Beclin1

Figure S6C

$\mathrm{C} 2+6 \% \mathrm{PI}(4,5) \mathrm{P} 2$
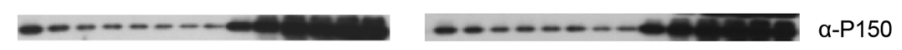
In addition, we are here to make a clarification about the negative controls in Supplementary information, Figs. S4E and S5F. In the manuscript, "C1" was identical to "WT" complex, which contains ATG14L, Beclin1, P150 and VPS34. "ATG14L $\triangle B A T s "$ complex was identical to "C1 $\triangle \mathrm{BATs}$ " complex, which contains ATG14L $\triangle$ BATs, Beclin1, P150 and VPS34. Different abbreviations came from particular experimental focuses. Also, some of the negative controls in Fig. 4E and Supplementary information, Fig. $\mathrm{S} 5 \mathrm{~B}$ were presented twice as they were generated from the same set of experiments. For clarification, we also updated figure legends for Supplementary information, Figs. S4E and S5F.

The correction does not affect the conclusion of this study.

MM from LY's lab performed all the related experiments and made figures. $M M$ and $L Y$ are responsible for all the above errors.

Figure S4. Membrane-binding orientation of C1.

e Flotation assay to test the interaction of C1 WT, C1 VPS34 $\triangle \mathrm{CTD}$ and $\mathrm{C} 1 \mathrm{ATG} 14 \mathrm{~L} \triangle \mathrm{BAT}$ s with liposomes containing $6 \% \mathrm{PI}$ in a sucrose gradient (from top to bottom: 0, 20\%, 25\%, 30\%). All 14 fractions from top to bottom were immunoblotted using antibodies against P150, VPS34 and Beclin1. The band of " $\mathrm{a}$ P150_ C1 $\triangle B A T s "$ was re-presented here (identical to Fig. 4E) because they were generated from the same set of experiment. "ATG14L $\triangle$ BATs" complex was identical to "C1 $\triangle$ BATs" complex, which contains ATG14L $\triangle$ BATs, Beclin1, P150 and VPS34.

Figure S5. The BATs domain determines the ER-binding capacity of $\mathrm{PI} 3 \mathrm{KC} 3$ complexes in vitro. $\mathrm{f}$ Flotation assay to test the interaction of $\mathrm{C} 1, \mathrm{C} 1 \triangle \mathrm{BATS}, \mathrm{C} 2$ and C2-BATs with the ER fraction in a sucrose gradient (from top to bottom: $0,20 \%, 25 \%$, $30 \%)$. All 14 fractions from top to bottom were analyzed using antibodies against VPS34, ATG14L, UVRAG and Beclin1. Because some negative controls were generated from the same set of experiment, the following bands were re-presented in this panel: "a-VPS34_C1" (identical to "a-VPS34_WT" in Fig. S4E), " $a$ Beclin1_C1 $\triangle$ BATs" (identical to "a-Beclin1_ATG14L $\triangle B A T s$ " in Fig. S4E), "a-UVRAG_C2-BATs" (identical to "a-UVRAG_C2-BATs" in Fig. S5B), "a-Beclin1_C2" (identical to "a-Beclin1_C2" in Fig. S5B) and "a-Beclin1_C2-BATs" (identical to "a-Beclin1_C2-BATs" in Fig. S5B). "C1" complex was identical to "WT" complex, both of which contain ATG14L, Beclin1, P150 and VPS34. "ATG14L $\triangle$ BATs" complex was identical to "C1 $\triangle$ BATs" complex, both of which contain ATG14L $\triangle$ BATs, Beclin1, P150 and VPS34. 